\title{
DIFFUSION NETWORKS FOR ON-CHIP IMAGE CONTRAST NORMALIZATION
}

\author{
Pietro Perona ${ }^{\dagger \dagger}$ and Marco Tartagni $\dagger \S$ \\ † California Institute of Technology 116-81, Pasadena, CA 91125 - perona@caltech.edu \\ ‡ Università di Padova, Italy \\ $\S$ Università di Bologna, Italy
}

\begin{abstract}
A new method for normalizing and quantizing images is presented. The method is based on calculating a local reference frame for the image gray levels. The levels of the reference frame are calculated using biased diffusions that are linked to the original image. The method is conceived to be integrated with sensing elements on the image plane of a camera. Its mathematical properties are analyzed and its performance is experimentally demonstrated.

A circuital implementation has been designed, constructed and tested; it consists of a 20 nodes 1-D non-linear resistive grid. Experimental results are shown.
\end{abstract}

\section{INTRODUCTION}

Consider the image in Fig. 1. It has been acquired indoors with a high-end commercial camcorder. The gain of the image sensor of the camera has been set automatically so as to capture as well as possible both the brightly lit and the darker areas in the scene. Unfortunately, due to the limited dynamic range of the sensor a 'perfect' gain setting is impossible: both the brighter and darker areas of the image are saturated. The human visual system solves this problem by allowing the gain to be different in different areas of the image: higher in regions of dim lighting and lower where the contrast is high. This way the information that is carried by the variations of contrast through the image is lost, however for the purpose of human image analyisi this appears to be irrelevant. In this paper we present a method of image contrast normalization that is built along similar lines. The contrast level is locally (implicitly) estimated and compensated for by a set of diffusive networks. The system that we propose may be implemented by means of simple electronic circuitry and may be embedded in the sensing circuitry of an electronic camera. A scheme presenting a different technical solution with the same functional characteristic has been previously proposed by Moore and collaborators [3].

The importance and difficulty of normalizing contrast in images are well known. A number of computational schemes have been proposed for normalizing automatically the contrast in images. The most popular are probably histogram equalization and homomorphic filtering (see any recent image processing textbook, e.g. [1], for a complete description). Histogram equalization consists in changing the colormap of the image with the monotonic transformation that transforms the histogram of the image gray levels into a constant. Homomorphic filtering is motivated by attributing all variations in image contrast to dishomogeneous lighting conditions; if one makes the hypothesis that such dishomogeneities are lowpass, then they may be eliminated by highpass

This work was supported by NSF Grant IRI 9306155 on "Geometry driven diffusions", by an NSF National Young Investigator Award and by scholarship 204.40.07 from the Consiglio Nazionale delle Ricerche of Italy.

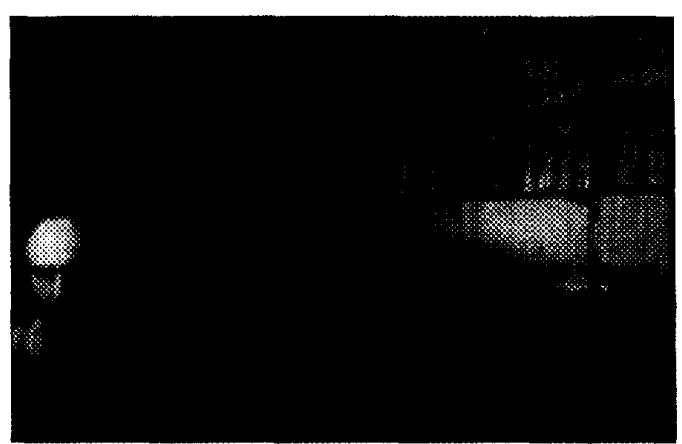

Figure 1:

filtering the $\log$ of the image since lighting contributes to the image as a multiplicative factor. Well-known difficulties with these techniques are that histogram equalization is too global and treats all areas of the image equally, while the lowpass hypothesis underlying homomorphic filtering is often false, and ringing patterns may be generated. A more detailed discussion of the pros and cons of these and other classical contrast normalization techniques goes beyond the scope of this paper.

\section{CONTRAST ESTIMATION AND IMACE NORMALIZATION}

It is difficult to give a precise and synthetic definition of what 'contrast normalization' exactly means. One possible approach, quite common in engineering, consists of three steps (a) concentrate one's attention on a subset of all possible images, deally this is a subset that is representative of the more general class of interest and for which a clear dedefinition of normalization $\mathrm{s}$ possible, (b) develop a normalization algorithm for the chosen subset, (c) experiment with and asses the algorithm on the general class of images of interest. As our 'working subclass' we chocse here the set of piecewise constant signals with a finite number of discontinuities that we will call 'edges' from now on. Since we wish to develop a local algorithm we must use a concept of 'sciale' $\lambda$ that will be a free parameter in the algorithm. The meaning of 'contrast normalization' is now easy to define: all isolated edges (i.e. further than one scale length from other edges ) should be considered significant, regardless their height, while edges which sre close by ( closer than one scale length) bigger edges should be considered insignificant and normalized to a punitive smaller height. 
Call $u$ the brightness function. We can summarize the properties that the normalization operation $N(\cdot)$ should have as follows:

- Causality: Edges of $u$ should correspond to edges of $N(u)$, and viceversa.

- Normalization: Edges after normalization should have height $\leq 1$. In particular isolated edges of $N(u)$ should have height $=1$.

- Invariance to scaling in the codomain: $N(\alpha \cdot u)=$ $\operatorname{sign}(\alpha) N(u)$. For $\alpha$ constant and different from zero.

- Invariance to offsets: $N(u+\alpha)=N(u)$. For $\alpha$ constant.

- Invariance to scaling in the domain: Calling $N_{\lambda}(u(\cdot))$ the normalization operator at a scale $\lambda$, and $s$ a positive scaling constant, then $N_{\lambda}$ should be such that $N_{\lambda}\left(u\left(\frac{1}{\cdot} \cdot\right)\right)(x)=$ $N_{\lambda}(u(\cdot))\left(\frac{1}{a} x\right)$

- Invariance to translations:

$N(u(\cdot-t))(x)=N(u(\cdot))(x-t)$

\subsection{Max-mean-min normalization}

The simplest way to build such a normalization map is to define $N(\cdot)$ as: $N(u)=\frac{u-u_{M}}{u_{T}-u_{B}}$ where $u_{m}$ (u-middle) is the average of $u$, and $u_{T}$ and $u_{B}$ (u-top, and u-bottom) the maximum and minimum of $u(\cdot)$ over the whole domain. It is easy to see that such a normalization satisfies the first, third, and fourth criterion.

For an image containing a single step edge this normalization would also satisfy the second criterion. In general this is not the case; the problem with this constant normalization is that it preserves the ratio of the contrast of the edges throughout the image, while we would like it to be normalized to 1 .

If the maximum, minimum, and average are computed instead on neighbourhoods of size equal to the scale at which we are looking at the image the second criterion can be satisfied. In other words $u_{T}=u_{T}(x), u_{M}=u_{M}(x)$ and $u_{B}=u_{B}(x)$ have to be local maximum, average, and minimum.

Not all methods to compute the three functions are good though: edges in $u_{T}(x), u_{M}(x)$ or $u_{B}(x)$ would introduce edges in the normalized function $N(u)$ violating the first requirement. In order not to introduce new "features" in the normalized image one has to ensure that $u_{T}(\cdot), u_{M}(\cdot)$ or $u_{B}(\cdot)$ are "smoother" than the features of $u(\cdot)$ one wants to detect. We will discuss next how to generate $u_{T}(x), u_{M}(x)$ or $u_{B}(x)$ that do not violate the first requirement.

Consider the functions $u_{S}(x)$ that minimize the following cost function :

$$
C(v)=\alpha_{S} d_{+}(u, v)+\beta_{S} d_{-}(u, v)+\lambda S(v)
$$

where $u$ is the function to be normalized, $S(v)$ measures the smoothness of $v, \lambda$ is a scale parameter determining the relative weight of the smoothness term in the cost function ( bigger $\lambda$ implies smoother minima of the cost function $), d_{+}(u, v)$ and $d_{-}(u, v)$ are the "distance" of $u$ from $v$ "from above" and "from below" i.e. are such that $d_{+}(u, v)+d_{-}(u, v)=d(u, v)$, $d(\cdot, \cdot)$ being some distance in $L^{2}$ and such that $d_{+}(u, v)=d(u, v)$ and $d_{-}(u, v)=0$ on the intervals where $u(x)>v(x)$, and that $d_{-}(u, v)=d(u, v)$ and $d_{+}(u, v)=0$ on the intervals where $u(x)<v(x)$. Notice that the choice of $d(\cdot, \cdot)$ determines uniquely $d_{+}(u, v)$ and $d_{-}(u, v)$

For all positive values of $\alpha$ and $\beta$ the mimimizer can be computed solving the corresponding Euler equation.
Call :

- $u_{M}$ the function minimizing the cost when $\alpha_{S}=1$ and $\beta_{S}=1$,

- $u_{T}$ the function minimizing the cost when $\alpha_{S}=\infty$ and $\beta_{S}=$ 1 ,

- $u_{B}$ the function minimizing the cost when $\alpha_{S}=1$ and $\beta_{S}=$ $\infty$

These functions can be seen respectively as :

- $u_{M}$ the $\lambda$-smooth function best approximating $u$,

- $u_{T}$ the $\lambda$-smooth function best approximating $u$ in the set $T(u)$ of functions above $u$, i.e. functions $v$ that satisfy $v \geq u$,

- $u_{B}$ the $\lambda$-smooth function best approximating $u$ in the set $B(u)$ of functions below $u$, i.e. the functions $v$ that satisfy $v \leq u$.

We shall take these three functions as local average, maximum and minimum.

If the smoothness function is appropriate the rest lting normalization will satisfy the desired properties. In order to satisfy the third and fourth requirement the smoothness term aas to be the integral of the square of a linear differential operato ; and the distance term has to depend only on the difference $(u-v)$ and be linear on the positive and negative semilines.

Take for example: $S(v)=\int\left(\frac{\partial v}{\partial x}\right)^{2} d x$ and $d(u v)=\int(u-$ $v)^{2} d x$. The cost function is convex for all positive vilues of $\alpha$ and $\beta$ and it's mimimizer can be computed solving the corresponding Euler equation

$$
0=\lambda \cdot \Delta v+f(u-v)
$$

where $f$ depends on the choice of $\alpha$ and $\beta$.

The solution of this kind of equation can be ites atively found by simulating the diffusion-type equation:

$$
\frac{\partial v}{\partial t}=\Delta v+\frac{1}{\lambda} f(u-v)
$$

where the choice of the function $f$ will determire the convercence to $u_{M}, u_{T}$, or $u_{B}$.

More in detail:

For $\alpha=\beta=1$ :

$$
\frac{\partial v}{\partial t}=\Delta v+\frac{1}{\lambda}(u-v)
$$

For $\alpha=\infty$, and $\beta=1$ :

$$
\frac{\partial v}{\partial t}(x)=\Delta v(x)+\frac{1}{\lambda}(u-v)(x)
$$

$\forall x$ at which $(u-v)(x)<0$, and

$$
v(x) \longrightarrow u(x)
$$

$a \forall x$ at which $(u-v)(x)>0$

For $\alpha=1$, and $\beta=\infty$ :

$$
\frac{\partial v}{\partial t}(x)=\Delta v(x)+\frac{1}{\lambda}(u-v)(x)
$$

$\forall x$ at which $(u-v)(x)>0$, and

$$
v(x) \longrightarrow u(x)
$$




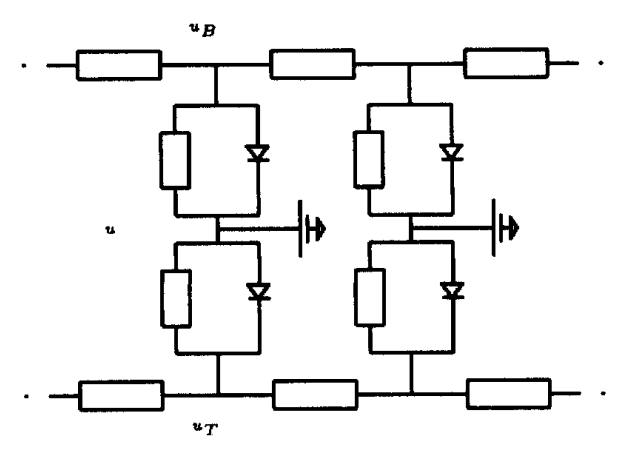

Figure 2: The structure of a diffusive network composed of ideal diodes and resistors, computing the top and bottom functions.

$\forall x$ at which $(u-v)(x)<0$

This kind of diffusion can be performed by the simple dioderesistor circuit shown in fig. 2.

It is simple to verify that $u_{T} \geq u \geq u_{B}$ and $u_{T} \geq u_{M} \geq u_{B}$ if the scale of the middle function $u_{M}$ is equal or finer ( smaller ) than the scale of the top and bottom functions $u_{T}$, and $u_{B}$.

\subsection{Local reference frame normalization}

In the previous section we have described a method for contrast normalization based on dividing the image by the difference of maximum and minimum. In this section we present a generalization of that method based on computing a full local frame of reference for the gray level values of the image.

Consider the cost function defined by Eq. (1). We have seen that by picking appropriate values for $\alpha$ and $\beta$ (namely 1 and $\infty$ ) we may obtain the mean, top and bottom functions (a formal proof is given in the next section).

If instead of picking only three discrete set of values for $\alpha$ and $\beta$ we may make $\alpha$ and $\beta$ functions of a parameter $\gamma$ so that when $\gamma=-1$ we have $\alpha(\gamma)=1$ and $\beta(\gamma)=\infty$, with $\alpha$ monotonically non-decreasing and $\beta$ non-increasing so that $\alpha(0)=1, \beta(0)=1$ and $\alpha(1)=\infty, \beta(1)=1$, this way we may generate a continuum of smooth functions ranging from the 'bottom', through the 'mean' to the 'top' function. This family of functions $u_{\gamma}$ (with $u_{-1}=$ bottom, $u_{0}=$ mean and $u_{+1}=$ top) will constitute $a$ frame of reference for the image $v$ in the following way:

Define $\gamma^{*}(x)$ the value of $\gamma$ such that $v(x)=u_{\gamma}(x)$; we may define the normalized image to be:

$$
N(v(x))=\gamma^{*}(x)
$$

Naturally this intuitive idea has sense only if the functions $u_{\gamma}$ are computable with a simple electrical network, are smooth and do not intersect, i.e. if $u_{\gamma_{1}}(x) \geq u_{\gamma_{2}}(x)$ for $\forall x$ and $\forall \gamma_{1} \geq \gamma_{2}$. In the next section we study a specific realization of the cost function that generates a family $u_{\gamma}$ having the desired properties. Some of the proofs are sketchy - for a detailed version see [4].

\subsection{Analysis}

Given a compact set $\Omega \subset \mathbf{R}^{n}$ (the image plane) and a bounded function $v: \boldsymbol{\Omega} \rightarrow \mathbf{R}$ (the image data) consider the 'energy' function $E$ defined on functions $u: \Omega \rightarrow \mathbf{R}$ as follows:

$$
E(u)=\lambda S(u)+d(v, u)
$$

where $\lambda \in(0, \infty), E$ is the $\lambda$-weighted sum of a 'sm sothness' term $S$ which assigns higher energy to functions $u$ that vary a lot, and a 'distance' term $d$ that assigns higher energy to fu uctions $u$ that are far from $v$. The definion of these functions based on $\mathrm{L}^{2}$ norms will be adopted here:

$$
\begin{aligned}
S(u) & =\int_{\Omega}|\nabla u(x)|^{2} d x \\
d(u, v) & =\int_{\Omega}|v(x)-u(x)|^{2} d x
\end{aligned}
$$

(notice that to simplify the notation what we call 'distance' is in fact the square of the usual $\mathrm{L}^{2}$-induced distance).

Define the $\lambda$-mean, the $\lambda$-maximum, and the $\lambda$-mini num of $v$ as the as the functions $\mu M m: \Omega \rightarrow \mathbf{R}$ that solve the iollowing minimization problems:

\section{Definition 1}

$$
\begin{aligned}
\mu & =\arg \min E(u) \\
M & =\arg \min _{u \geq v} E(u) \\
m & =\arg \min _{u \leq v} E(u)
\end{aligned}
$$

Define the ' $\gamma$-skewed parabola' $F_{\gamma}(\cdot)$ for $\gamma \in(-1,1)$ in the following way:

Definition 2

$$
F_{\gamma}(\cdot)=f_{-}(\gamma)(\cdot)_{-}^{2}+f_{+}(\gamma)(\cdot)_{+}^{2}
$$

where $(\cdot)_{+}$and $(\cdot)_{-}$denote the positive- and negative- $e_{-}$art functions, and where the $f_{ \pm}$satisfy the following requirements:

$$
\begin{aligned}
& \text { I. } f_{+}:(-1,1) \rightarrow(1,+\infty) \\
& \text { 2. } \gamma_{1}<\gamma_{2} \Rightarrow f_{+}\left(\gamma_{1}\right) \leq f_{+}\left(\gamma_{2}\right) \\
& \text { 3. } f_{+}(\gamma)=1 \text { when }-1 \leq \gamma \leq 0 \text {, } \\
& \text { 4. } f_{+}(\gamma \rightarrow 1)=+\infty, \\
& \text { 5. } f_{-}(\gamma)=f_{+}(-\gamma)
\end{aligned}
$$

Notice that $F_{\gamma}(\cdot)$ is constructed from the standarc parabola $(\cdot)^{2}$ in the following way: when $-1<\gamma<0$ the left branch of the parabola is 'raised' by multiplication with $f_{-}>1$, the more so the bigger $|\gamma|$, while when $0<\gamma<1$ the left branch of the parabola is raised by multiplication with $f_{+}>1$. Therefore the skewed parabola $F_{\gamma}(w)$ is monotonically nonincreasing in $\gamma$ for $w<0$ and monotonically nondecreasing for $w>0$. lows:

Using $F_{\gamma}$ define a ' $\gamma$-distance' $d_{\gamma}$ between functions as fol-

$$
d_{\gamma}(u, v)=\int_{\Omega} F_{\gamma}(u-v) d x
$$

Notice that $d_{\gamma=0}(u, v)=d(u, v)$; moreover $d_{\gamma=1}(u, v)=$ $d(u, v)$ if $u \geq v$ while it is $\infty$ if on any set of nonzero measure 

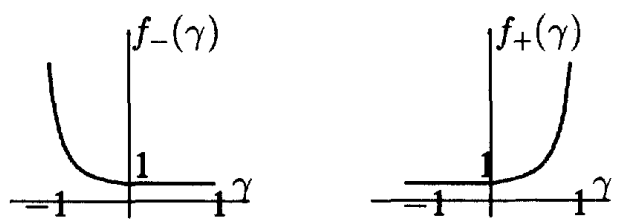

Figure 3: The weighting functions $f_{-}(\gamma)$ and $f_{+}(\gamma)$.
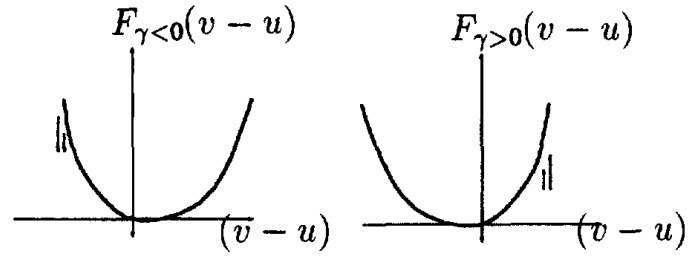

Figure 4: The modified parabola $F_{\gamma}(v-u)$.

$u<v$. Similarly for $d_{\gamma=-1}$ with the appropriate inequality sign inversions. in (3):

Using the new distance $d_{\gamma}$ define a new energy function $E_{\gamma}$ as

$$
E_{\gamma}(u)=\lambda S(u)+d_{\gamma}(u, v)
$$

Define the $\lambda-\gamma$-mean $u_{\gamma}$ of $v$ as the minimum of $E_{\gamma}$ :

Definition 3

$$
u_{\gamma}=\arg \min E_{\gamma}(u)
$$

The behaviour of $u_{\gamma}$ is described by the following propositions:

Proposition $1 u_{\gamma}$ is the unique solution of the differential equation:

$$
\left\{\begin{array}{lr}
\lambda \Delta u-f_{-}(\gamma)(u-v)_{-}+f_{+}(\gamma)(u-v)_{+}=0 & x \in \Omega \\
\nabla u=0 & x \in \partial \Omega
\end{array}\right.
$$

where the gradient and laplacian are taken with respect to the 'space' variables $x$.

\section{Proof:}

The energy function (9) is convex, therefore it has a unique minimum. The differential equation (11) is the Euler equation of the variational problem (10).

ㅁ.

Proposition 2 The functions $u_{\gamma}$ are ordered with respect to $\gamma$, i.e. if $\gamma_{1}<\gamma_{2}$ then $u_{\gamma_{1}}(x) \leq u_{\gamma_{2}}(x)$ for all $x \in \Omega$ with the exception of zero-measure subsets of $\Omega$.

Proof:

By contradiction, showing that if $u_{\gamma_{1}}(x)>u_{\gamma_{2}}(x)$ in a subset $I$ of $\Omega$ then one may decrease the sum of the energies by swapping $u_{\gamma_{1}}$ and $u_{\gamma_{2}}$ in $I$ since restricting ourselves to the set $I$ we have $E_{\gamma_{1}}\left(u_{\gamma_{1}}\right)+E_{\gamma_{2}}\left(u_{\gamma_{2}}\right)>E_{\gamma_{1}}\left(u_{\gamma_{2}}\right)+E_{\gamma_{2}}\left(u_{\gamma_{1}}\right)$.

Detailed argument:

Suppose that $u_{\gamma_{1}}(x)>u_{\gamma_{2}}(x)$ in a subset $I$ of $\Omega$. Partition $I$ in the subsets $A 1, A 2, B, C$ such that: $u_{\gamma_{1}}(x) \geq v(x)>u_{\gamma_{2}}(x)$ for $x \in A 1$,

$u_{\gamma_{1}}(x)>v(x) \geq u_{\gamma_{2}}(x)$ for $x \in A 2$,

$u_{\gamma_{1}}(x)>u_{\gamma_{2}}(x)>v(x)$ for $x \in B$ and

$v>u_{\gamma_{1}}(x)>u_{\gamma_{2}}(x)$ for $x \in C$

For convenience of notation define the functions $w_{\gamma}=u_{\gamma_{i}}-v$. Now compare the distances on the subsets:

A1, A2 - In $A 1 \quad w_{\gamma_{1}} \geq 0>w_{\gamma_{2}}$, therefore $d_{\gamma_{1}}\left(w_{\gamma_{1}}\right) \geq$ $d_{\gamma_{1}}\left(w_{\gamma_{2}}\right)$ and $d_{\gamma_{2}}\left(w_{\gamma_{2}}\right)>d_{\gamma_{2}}\left(w_{\gamma_{1}}\right)$. The same happens in $A 2$ modulo exchanging equality and inequality signs.

B, C - In $B w_{\gamma_{1}}>w_{\gamma_{2}}>0$. Call $d_{\delta \gamma}==d_{\gamma_{1}}-d_{\gamma_{2}}$ Observe that for positive argument $d_{\delta \gamma}$ is positive and monotonically increasing. Then $d_{\gamma_{1}}\left(w_{\gamma_{1}}\right)+d_{\gamma_{2}}\left(w_{\gamma_{2}}\right)=d_{\gamma_{2}}\left(w_{\gamma_{1}}\right)+$ $d_{\delta \gamma}\left(w_{\gamma_{1}}\right)+d_{\gamma_{2}}\left(w_{\gamma_{2}}\right)>d_{\gamma_{2}}\left(w_{\gamma_{1}}\right)+d_{\delta \gamma}\left(w_{\gamma_{2}}\right)+d_{\gamma_{2}}\left(w_{\gamma_{2}}\right)=$ $d_{\gamma_{2}}\left(w_{\gamma_{1}}\right)+d_{\gamma_{1}}\left(w_{\gamma_{2}}\right)$. The same may be shown in $C$ noticing that $0>w_{\gamma_{1}}>w_{\gamma_{2}}$ and that for negative arguments $d_{i \gamma}$ is negative and monotonically decreasing.

Following the derivations in the previous two paragraphs notice that by swapping $u_{\gamma_{1}}$ and $u_{\gamma_{2}}$ in $I$ all terms in the sum of the energy functions are unchanged with the exception of the distances $d_{\gamma_{1}}$ and $d_{\gamma_{2}}$ that decrease on $I$. Therefore contradicti on is reached.

口.

Lastly we show that $M$ and $m$ are the upper ancl lower limits of the functions $u_{\gamma}$.

We start by showing that at the minimum $u_{\gamma}$ the energy function $E_{\gamma}$ tends to $E$ for $\gamma \rightarrow \pm 1$

Proposition 3

$$
\lim _{\gamma \rightarrow \pm 1} E_{\gamma}\left(u_{\gamma}\right)=E\left(u_{\gamma}\right)
$$

\section{Proof:}

The proof is carried out for $\gamma \rightarrow 1$. The same technique may be used to prove the $\gamma \rightarrow-1$ limit.

By definition of $u_{\gamma}$ (see (10)) $\forall \delta u$ s.t. $\delta u: 2 \rightarrow \mathbf{R}$ and $\delta u=0$ on $\partial \Omega$ :

$$
\lim _{h \rightarrow 0} \frac{E_{\gamma}\left(u_{\gamma}+h \delta u\right)-E_{\gamma}\left(u_{\gamma}\right)}{h}=0
$$

This is true in particular for a constant variation $5 u \equiv 1$.

The gradient of $\delta u \equiv 1$ is of course equal to zero. Expand $E_{\gamma}\left(u_{\gamma}+h \cdot 1\right)$ around $E_{\gamma}\left(u_{\gamma}\right)$ using for convenience $h \geq 0$ :

$$
\begin{array}{r}
E_{\gamma}\left(u_{\gamma}+h \cdot 1\right)=0\left(h^{2}\right)+ \\
E_{\gamma}\left(u_{\gamma}\right)+2 h \int_{\Omega} f_{+}(\gamma)\left(u_{\gamma}-v\right)_{+}-f_{-}(\gamma)\left(u_{\gamma}-\imath\right)_{-} d x+ \\
+\int_{\Omega \pm}\left[f_{+}(\gamma)-f_{-}(\gamma)\right]\left[(u-v)_{-}^{2}+2 h(u-v)_{-}\right] d x
\end{array}
$$

where $\Omega \pm$ is the subset of $\Omega$ on which $-h<(u-v)<0$. Notice that the integral on $\Omega \pm$ is $O\left(h^{2}\right)$ since by hyp. $\Omega$ has finite measure $\mu(\Omega)$ (and therefore $\Omega \pm$ also has finite measure), and, by definition of $\Omega \pm$ the upper bound of $|u-v|$ on $\Omega \pm$ is $h$. An upper bound for the modulus of the integral is therefore $\left|f_{+}(\gamma)-f_{-}(\gamma)\right| \mu(\Omega) h^{2}$. 


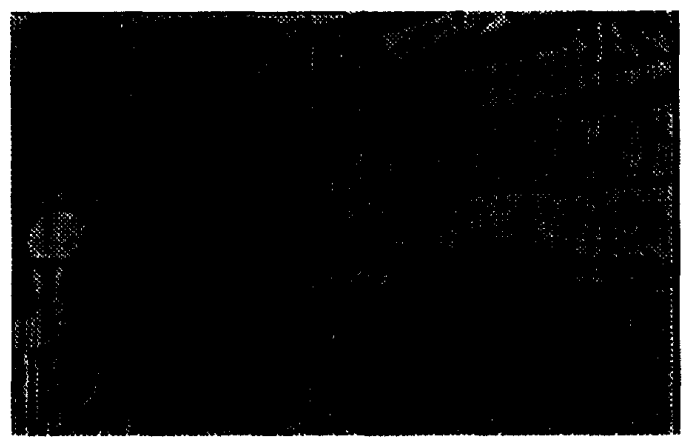

Figure 5: Normalization and quantization to 3 bits using 8 diffusive levels with $\lambda=10$ pixels of original in Fig. 1.

Equation (15) therefore reduces to:

$$
\begin{array}{r}
E_{\gamma}\left(u_{\gamma}+h \cdot 1\right)=O\left(h^{2}\right)+ \\
E_{\gamma}\left(u_{\gamma}\right)+2 h \int_{\Omega} f_{+}(\gamma)\left(u_{\gamma}-v\right)_{+}-f_{-}(\gamma)\left(u_{\gamma}-v\right)_{-} d x
\end{array}
$$

From (13) and (16) we see that for $\forall \gamma$

$$
f_{+}(\gamma) \int_{\Omega}\left(u_{\gamma}-v\right)_{+} d x=f_{-}(\gamma) \int_{\Omega}\left(u_{\gamma}-v\right)_{-} d x
$$

Now notice that $\left(u_{\gamma}-v\right)_{+}$has an upper bound $M$ in $\Omega$ (e.g. clearly $\left(u_{\gamma}-v\right)_{+}\langle\max (v)-v)$. Moreover by definition for $\gamma>$ $1 f_{+}(\gamma) \equiv 1$. Therefore $C=M \mu(\Omega) \geq f_{+}(\gamma) \int_{\Omega}\left(u_{\gamma}-v\right)_{+} d x$, the 1.h.s. of (16). Squaring both terms and dividing both by $f_{-}(\gamma)$ (which is bounded away from zero) we obtain:

$$
\begin{aligned}
f_{-}^{-1}(\gamma) C \geq f_{-}(\gamma) & \left(\int_{\Omega}\left(u_{\gamma}-v\right)_{-} d x\right)^{2} \geq \\
& \geq \int_{\Omega} f_{-}(\gamma)\left(u_{\gamma}-v\right)_{-}^{2} d x
\end{aligned}
$$

Clearly $\lim _{\gamma \rightarrow 1} f_{-}^{-1}(\gamma) C=0$ and therefore

$\lim _{\gamma \rightarrow 1} \int_{\Omega} f_{-}(\gamma)\left(u_{\gamma}-v\right)_{-}^{2} d x=0$. This proves that $\lim _{\gamma \rightarrow 1} E_{\gamma}\left(u_{\gamma}\right)=$ $E\left(u_{\gamma}\right)$.

ㅁ.

Proposition 1 shows that the normalization functions may be computed using a network of the type shown in Figure 6.

\section{HARDWARE IMPLEMENTATION}

A 20 nodes 1-D non-linear resistive grid has been implemented in a MOSIS tiny-chip and tested. Static input patterns are externally set by analog pads while the output pattern is scanned out by a static shift register [2] and read-out by an oscilloscope. Results are shown in Fig. 7 where several output patterns are compared with input data. Gamma values are displayed in each picture. If we disregard the noise introduced by the scanner, it is apparent how the circuit displays full functionality providing a complete family of outputs, bounded by an envelope of local maxima and an envelope of local minima, where "local" is defined by the diffusion lenght $\lambda$. Full details on the circuit are reported in [4].

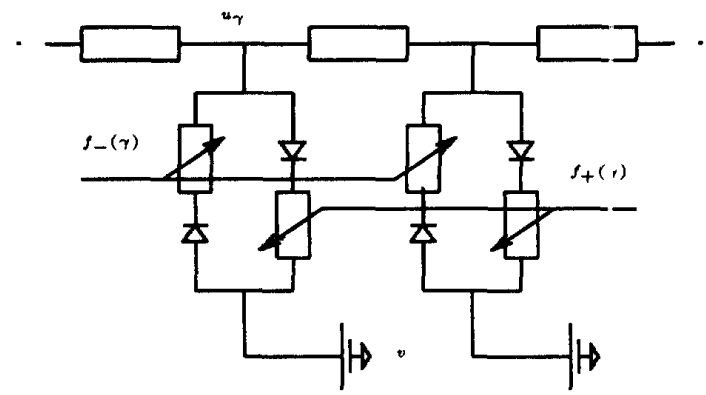

Figure 6: The structure of a diffusive network compossd of ideal diodes and resistors, computing the top and bottom functions.

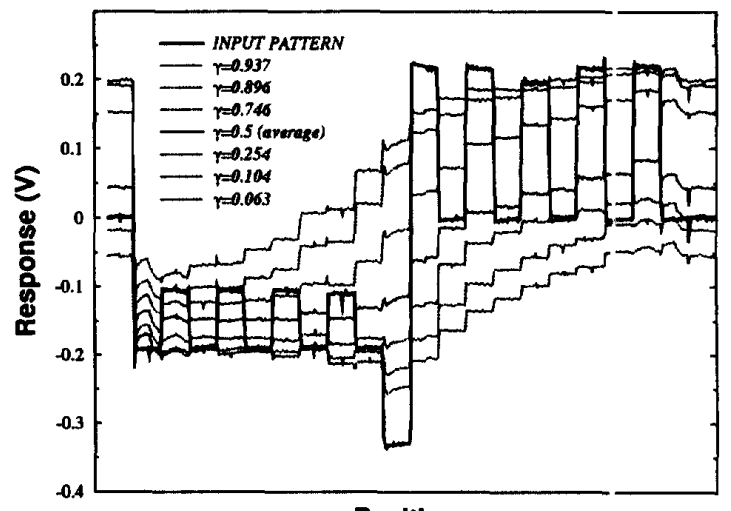

Position

Figure 7: Experimental data of output voltage response are plotted versus node position for different values of $\gamma$ and using a small diffusion length

\section{ACKNOWLEDGEMENTS}

We are very grateful to Gabriella Tarantello, Jitendra Malik, Jean-Michel Morel, David Mumford, Michele Payon and Sergio Solimini for discussions concerning the proofs of the theorems.

\section{REFERENCES}

[1] A. Jain. Fundamentals of digital image processing. Infermation and systems sciences. Prentice Hall, 1989.

[2] C. Mead and T. Delbruck. Scanners for visualizing activ ty of analog vlsi circuitry. In AICSP1, pages 93-106, 1991.

[3] A. Moore, J. Allman, and R. Goodman. A real-time numral system for color constancy. IEEE Trans. on Neural Networks, 212):237-247, 1991.

[4] P. Perona and M Tartagni. Contrast normalization and ims ge quantization using biased diffusions. Computation and neural syste ms technical report, California Institute of Technology, 1994. In prepacation. 